\title{
Graph Based Interactive Detection of Curve Structures in 2D Fluoroscopy
}

\author{
Peng Wang, Wei-shing Liao, Terrence Chen, Shaohua K. Zhou, \\ and Dorin Comaniciu
}

Siemens Corporate Research, 755 College Road East, Princeton NJ, U.S.A.

\begin{abstract}
An accurate and robust method to detect curve structures, such as a vessel branch or a guidewire, is essential for many medical imaging applications. A fully automatic method, although highly desired, is prone to detection errors that are caused by image noise and curve-like artifacts. In this paper, we present a novel method to interactively detect a curve structure in a $2 \mathrm{D}$ fluoroscopy image with a minimum requirement of human corrections. In this work, a learning based method is used to detect curve segments. Based on the detected segment candidates, a graph is built to search a curve structure as the best path passing through user interactions. Furthermore, our method introduces a novel hyper-graph based optimization method to allow for imposing geometric constraints during the path searching, and to provide a smooth and quickly converged result. With minimum human interactions involved, the method can provide accurate detection results, and has been used in different applications for guidewire and vessel detections.
\end{abstract}

\section{Introduction}

In medical imaging, an accurate and robust method to detect curve structures, such as a vessel branch in angiogram, or a guidewire or a catheter in fluoroscopy, is required in many practical applications. Although a fully automatic method is always highly desired, current automatic methods are prone to the errors that are caused by image noise, and curve-like artifacts. In some applications where a large error is not tolerated, human interactions are introduced to make corrections. There are also some cases where human interactions are needed to specify a curve structure of particular interests. For examples, clinicians need to specify a vessel branch where interventions will take place, or users are more interested in a guidewire instead of a catheter, as shown in Figure 1 Such situations highlight the necessity to have interactions in practical systems. In this paper, we present a novel method based on a graph optimization to interactively detect a curve structure in a $2 \mathrm{D}$ fluoroscopy image with a minimum requirement of human corrections.

There are existing methods to automatically detect curve structures, such as guidewire 1], and vessels [2], while less research has been done on the interactive curve detection. Some existing interactive detection and segmentation methods 34. apply a graph cut algorithm, which needs user inputs to initial positive

T. Jiang et al. (Eds.): MICCAI 2010, Part III, LNCS 6363, pp. 269-277, 2010.
(C) Springer-Verlag Berlin Heidelberg 2010 


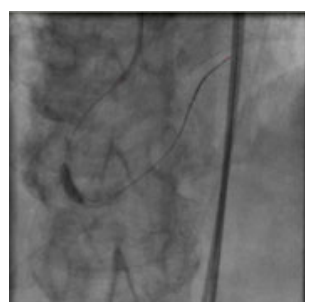

(a)

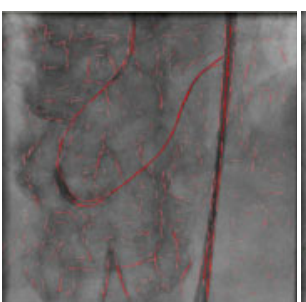

(b)

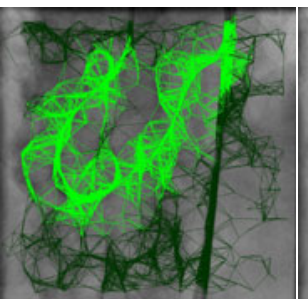

(c)

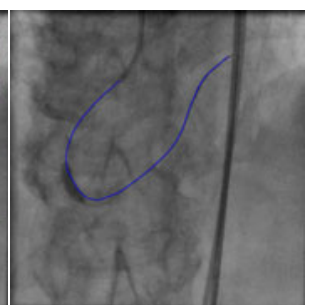

(d)

Fig. 1. Graph based interactive detection. (a) an original frame; (b) detected curve segments shown on red; (c) an original graph shown in dark green, and the second stage graph shown in bright green; (d) an interactively detected guidewire in blue.

and negative samples. However, the graph cut based methods mainly aim at the region segmentation, while in 2D fluoroscopy, the curve structure such as guidewire can be thin. The work 5 by Mazouer and his colleagues is close to the work in this paper. It detects guidewire segments in a hierarchical manner, and then finds the guidewire by minimizing the connectivity cost between segments using Dynamic Programming. However, the method only considers the pair-wise segment connectivity, and ignores the curve geometry constraints. When the image quality of 2D fluoroscopy is poor, the method is prone to image noises, and cannot converge to a satisfactory accuracy. It is shown in [5] that false detection rate drops to $4 \%$ after 7 user clicks, but there are no further improvements even given more user clicks.

To address the aforementioned problems, this paper presents a novel graph based framework and a hyper-graph based extension for interactive detection. The method automatically detects curve segments, which, together with user interactions, are used to construct a graph. In the graph, the nodes represent the curve segments, and the edges represent the curve connectivity. The method then formalizes the curve detection as to find the shortest path in the graph given user inputs. To impose curve geometric constraints, our method introduces a hypergraph, where the geometric constraints can be imposed as an inherent property. The presented work integrates the automatically detected curve segments, user interactions, and generic geometric constraints into one framework, and provides a smooth and quickly converged detection result. Extensive experiments demonstrate that with minimum human interactions involved, the method can detect various curve structures, such as guidewire and vessels, in 2D fluoroscopic images.

\section{Interactive Detection Based on Graph Optimization}

The presented interactive method consists of three components: automatically detecting curve segments, constructing a graph based on automatic detections and user interactions, and detecting a curve based on graph optimization. In this section, we first introduce the framework based on a conventional graph, and then extend it based on a hyper-graph in the next section. 


\subsection{Hierarchical Learning-Based Curve Segment Detection}

The method adapts a hierarchical learning based method that is similar to the method presented in the work [1. One difference is that the detectors used in this method only aim at detecting curve segments, not a whole curve. The detection includes two steps: first to detect piece-wise curve segments, and then to detect pair-wise connections between two curve segments. In the first step, the piece-wise segment detector detects a single piece of curves, namely a short line segment with a constant length. Such a curve segment has three parameters $(x, y, \theta)$, where $(x, y)$ is the segment center location and $\theta \in[-90,90]$ is the segment orientation. Such a learning based detector is trained from offline collected data. All the points on the annotated curves are considered positive training samples, while negative samples are randomly obtained from the regions containing no curve structures. The piece-wise segment detector uses Haar features [6] and the PBT classifier [7]. To detect segments of different orientations, an image is rotated at discrete angles to search the rotation angles of curve segments. Figure 1(b) shows detected piece-wise curve segments of a guidewire.

Since the piece-wise detector usually produces a lot of false alarms, a pairwise curve segment detector is used to prune false detections. In the pair-wise segment detection, every pair of two curve segments are classified if they belong to a curve, based on the image intensity and geometric features. Such geometric features include the center distance and angle difference between two segments. The same as the piece-wire segment detector, a PBT classifier is used to train the pair-wise curve segment detector. The probabilistic output form the pair-wise segment detector, noted as $p_{i, j}$, quantifies the connectivity between the $i$-th and $j$-th curve segments.

\subsection{Graph Representation for Interactive Detection}

In this method, a graph is used to organize all the detected curve segments, and the curve structure detection is then formalized as an optimization problem based on the graph. As shown in Figure2, (a), each node in a graph represents a detected segment, and an edge between two nodes represents a possible connection between them. We denote the node as $n_{k}$ for the $k$-th segment, and denote

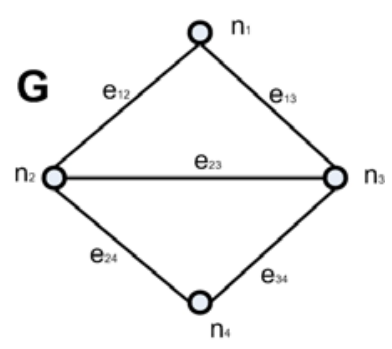

(a)

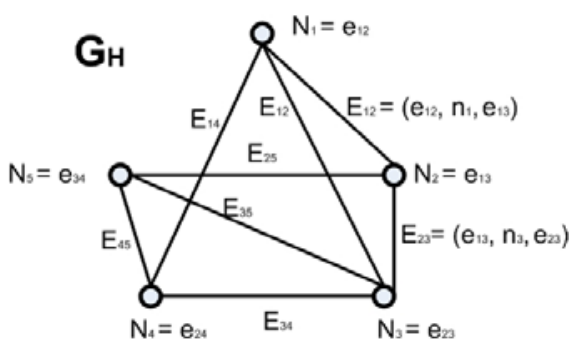

(b)

Fig. 2. Graph representation for curve detection (a) an original graph; (b) a hypergraph 
the edge as $e_{i j}$ for the detected connection between $i$-th and $j$-th node. Each edge $e_{i j}$ is associated with a cost $c_{i j}$, while the cost $c_{k}$ associated with the $k$-th node is usually ignored in the original graph.

The edge cost in our method is defined by the probabilistic outputs of the pair-wise detector, because it quantifies the connection between two nodes. Here we define the cost of an edge as in Eqn. (11):

$$
c_{i j}=-\log \left(p_{i, j}\right) .
$$

By this definition, the higher the probability is, the lower the cost is. Such a definition allows for formalizing the interactive detection as an optimization problem in the graph, as in Eqn. (2):

$$
\Gamma=\underset{L}{\arg \min } \sum_{(i, j) \in L} c_{i, j}=\underset{L}{\arg \max } \sum_{(i, j) \in L} \log \left(p_{i, j}\right),
$$

where $\Gamma$ is the detected curve, and $L$ is a valid path in the graph. Therefore, the curve detection is to find a best path in the graph, given the edge cost defined as in Eqn. (1). Given two end points on the curve, one as the source and the other as the destination node, the curve detection can be solved by a classical shortest path algorithm, such as the Dijkstra's algorithm [8|9] used in this method.

In the interactive detection, the source and destination nodes are specified by the first two user clicks, e.g., the first input point as the source node, and the second as the destination node. Each user click will create a new curve segment, whose position is defined by the user click, and whose orientation is interpolated from neighboring detected segments using the extension field [10]. The source and destination segments generated from user clicks are then used in the shortest path computations. After the first two clicks, additional user clicks may be needed to improve an interactive detection result. The additional user interactions are used to generate new curve segments in the same way as mentioned above. The user interaction continues until a satisfactory result is obtained.

\section{Extension to Hyper-Graph Based Optimization}

The graph optimization provides a solid framework for interactive detections. However, the best path solved in Eqn. (2) only involves the minimization of costs based on the classification scores from the pair-wise segment detector. Geometric properties of the curves, such as curve smoothness, have not been taken into considerations. Without geometric constraints, the detected curves can easily be distracted by false detections, and could generate unwanted results. A problematic detection result is shown in Figure 3. (b). Such path-related curve smoothness constraints are difficult to be incorporated in the conventional graph optimization algorithms, such as Dijikstra's. To address this issue, our method further extends an original graph to a hyper-graph which can accommodate geometric constraints in the detection framework.

The basic idea of constructing a hyper-graph is to model a segment of curve path, which involves more than a pair of nodes, as a hyper-edge (an edge in the 
hyper-graph, denoted as $\left.E_{i, j}\right)$, and also to model an edge in the original graph as a hyper-node (a node in the hyper-graph, denoted as $N_{k}$ ) in the hyper-graph. A simple example is shown as in Figure 2. An original graph $\mathbf{G}$ is shown in Figure 2. (a), and its corresponding hyper-graph $\mathbf{G}_{H}$ is shown in Figure 2.(b). The hyper-node $N_{i}$ is transformed from one of original graph's edges $e_{j, k}$, and a hyper-edge $E_{i, j}$ in $\mathbf{G}_{H}$ is transformed from a path $\left(e_{m, n} \rightarrow n_{m} \rightarrow e_{m, l}\right)$ in $G$, where $j, k, m, n, l$ are indices. In this way, a hyper graph is constructed from an original graph, and such a graph transformation is unique and reversible.

In the hyper-graph, a hyper-node inherits the edge cost in the original graph, i.e. $C_{N_{k}}=c_{i j}$ if $N_{k}$ corresponds to $e_{i j}$. For the cost of a hype-edge, since a hype-edge corresponds to a part of path on a curve, the cost of the hype-edge is then defined to impose the desired geometric constraints. In this method, two types of geometric constraints, shortness and the smoothness, are used to define the edge cost as in Eqn. (3):

$$
C_{E_{(i, j)}}=\alpha\left(1+\cos \left(\angle\left(E_{(i, j)}\right)\right)\right)+\beta \log \left(\frac{1}{1+\exp \left(-\mid\left(E_{(i, j)} \mid / B\right)\right.}\right),
$$

where $\angle\left(E_{(i, j)}\right)$ is the angle at the center of curve path that corresponds to the hyper-node, and $\left|E_{(i, j)}\right|$ is the arc length of the path. The first term $1+$ $\cos \left(\angle\left(E_{(i, j)}\right)\right)$ imposes the non-negative smoothness measurements of a curve path, and the second term $\log \left(\frac{1}{1+\exp \left(-\left|E_{(i, j)}\right| / B\right)}\right)$ favors a shorter curve. The parameters $\alpha$ and $\beta$ balance the two types of geometric constraints, and $B$ is a parameter in the sigmoid function. These parameters are empirically set in this method.

Given the constructed hype-graph and the redefined costs on edges and nodes, a graph shortest path algorithm finds the optimal curve, based on the criteria form both pair-wise connectivity and geometric constraints, as in Eqn. (4):

$$
\Gamma=\underset{L}{\arg \min } \sum_{N_{k} \in L \& E_{(i, j)} \in L} C_{N_{k}}+C_{E_{(i, j)}}
$$

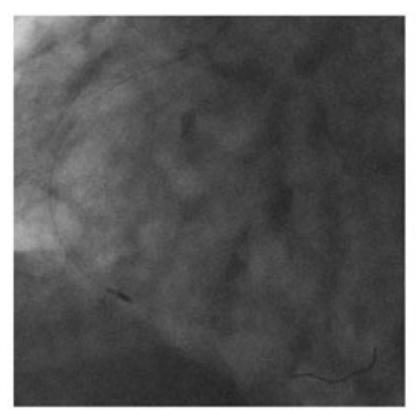

(a)

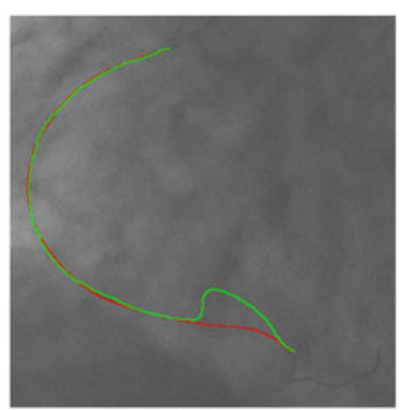

(b)

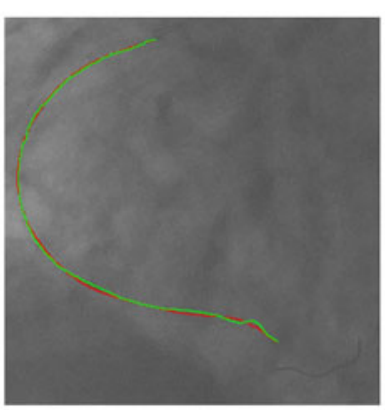

(c)

Fig. 3. Using hyper-graph to impose geometric constraints. (a) a frame; (b) the result based on the original graph; (c) the result based on the hyper-graph. The green lines represent interactive detections, and red lines are annotations. 
- Given the original graph $\mathbf{G}$, the source node $n_{s}$, and the destination node $n_{d}$

- Construct a hyper-graph $\mathbf{G}_{H}$

1. Set each edge in $\mathbf{G}$ as a hyper-node in $\mathbf{G}_{H}$, and copy the edge cost in $\mathbf{G}$ as a node cost in $\mathbf{G}_{H}$

2. Connect two hyper-nodes if they share a node in $\mathbf{G}$, and compute the hyperedge cost as in Eqn. (3)

- Find the shortest path in the hyper-graph $\mathbf{G}_{H}$

1. Get a set of edges $\left\{e_{i, s}\right\}$ that connect to $n_{s}$ in $\mathbf{G}$, and a set of edges $\left\{e_{j, d}\right\}$ that connect to $n_{d}$ in $\mathbf{G}$.

2. Compute a bundle of shortest distance. Each shortest distance is for a pair of hyper-nodes in $\mathbf{G}_{H}$. In each pair, one hyper-node corresponds to one of $\left\{e_{i, s}\right\}$, and the other corresponds to one of $\left\{e_{j, d}\right\}$

3. Select the optimal path from the bundle of shortest pathes

4. Map the shortest path in $\mathbf{G}_{H}$ back to the original path in $\mathbf{G}$

Fig. 4. Algorithm of hyper-graph based curve detection

Slightly different from the original graph, the single-source shortest path algorithm needs to be run multiple times to find a bundle of shortest paths in the hyper-graph, because each source node in the original graph corresponds to multiple edges in the hyper-graph, as shown in Figure 2 . From the bundle of shortest paths, an optimal curve with the least cost is selected as the final detection result. Figure 4 summarizes the hyper-graph based detection algorithm. Figure 3 shows an example of the improved detection of using hyper-graph. The numerical evaluations are further conducted in Section 4.

When constructing a hyper-graph from an original graph, the size of hypergraph grows linearly with the number of edges and nodes in the original graph. With the increased graph size, the computation time increases drastically. To address the computation problem, a two-stage method is used. In the two-stage method, the original graph and the hyper-graph methods are applied sequentially. In the first stage, a shortest path is found in the original graph. The curve segments that are far way from the initial detection are removed, therefore the hyper-graph built in the second stage is more compact. By using this two-stage detection strategy, the method can enjoy both the fast computation in the original graph and the benefits of geometric constraints in the hyper-graph. Figure 11.(c) and (d) show an example of the two-stage detection.

\section{Evaluations}

The presented interactive method can be applied to detect various curve structures, such as catheters, vessels, and guidewires in 2D fluoroscopy. For the quantitative evaluations, we select a set of 500 frames over 50 fluoroscopic sequences, which mainly contain guidewires. The image set includes challenging scenes for curve detection, such as various shapes and low signal-to-noise ratio. Some of testing images and results are shown in Figure 5](a). All the wires in the 

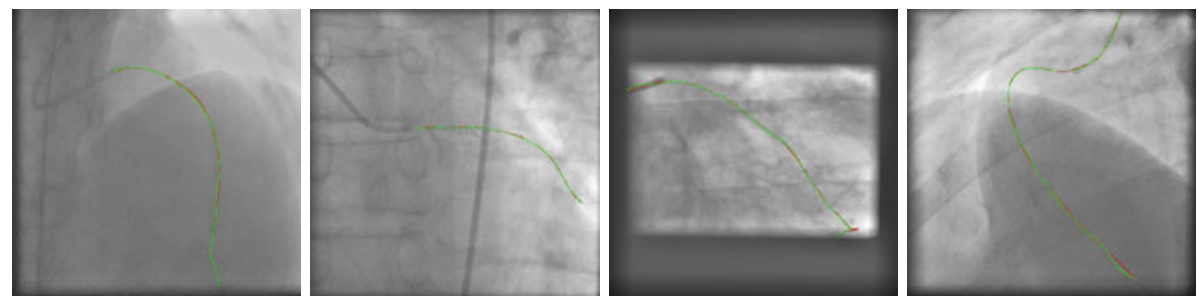

(a)
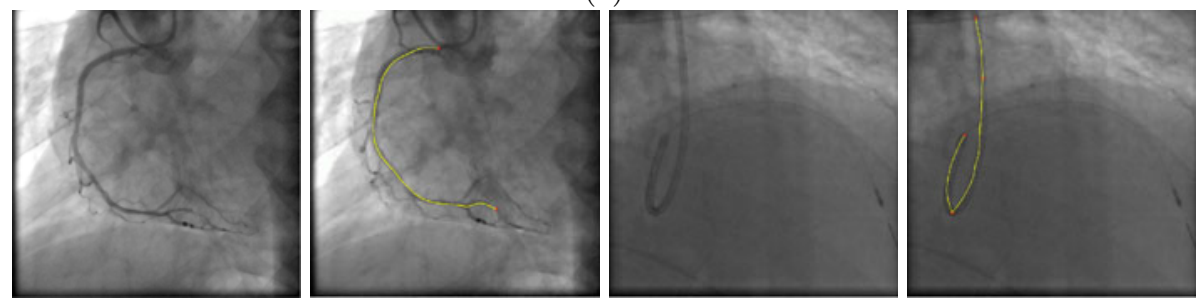

(b)

(c)

Fig. 5. Exemplar interactive detection results. (a) detected guidewires, where the green lines represent interactive detections with only two clicks, and red lines are annotations; (b) an image with a vessel branch in it, and the interactive detection result; (c) an image with a guiding catheter in it, and the interactive detection result.
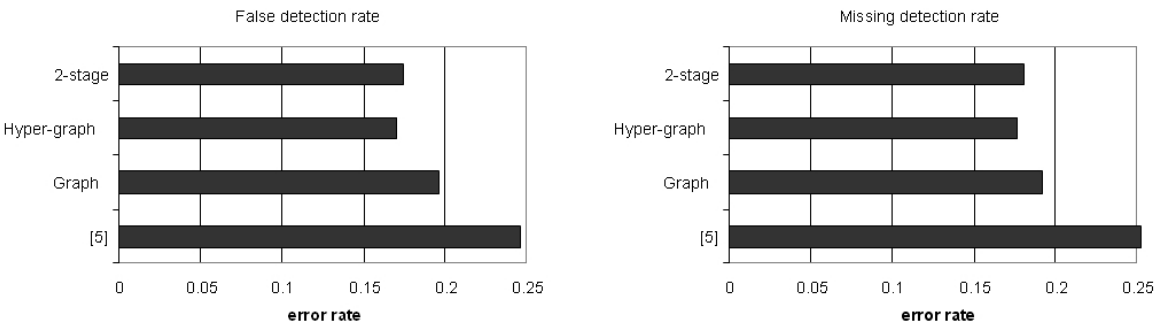

Fig. 6. Accuracy comparison of different methods with only 2 clicks

sequences are manually annotated as the ground truth. To measure the interactive detection accuracy, we compute two quantities: the false detection rate, and the missing detection rate. The false detection rate is the percentage of false detections that are at the distance of at least 3 pixels away from annotations. The missing detection rate is the percentage of annotated pixels that are at the distance of at least 3 pixels away from detection curves. We also measure the mean, standard deviation, and median of the distance between the detection results and annotation results. The experiments are conducted on a dual core $2.4 \mathrm{GHz}$ Desktop PC with $3 \mathrm{~GB}$ of RAM.

In the first experiment setting, only two user clicks are provided, and the detection accuracy is measured under the minimum interaction. Figure 6] and Table 1 show the accuracy comparison between different methods with only two 
Table 1. Quantitative evaluations on interactive detections with only two clicks

\begin{tabular}{|l|c|c|c|c|}
\hline & \multicolumn{2}{|c|}{ Detection Error (in pixels) } & Running time (in seconds) \\
\hline & median & mean & std & (excluding segment detections) \\
\hline$\underline{5}$ & 10.7 & 18.7 & 6.7 & 0.19 \\
\hline Graph & 8.3 & 15.1 & 6.7 & 0.02 \\
\hline Hyper-Graph & 6.8 & 12.5 & 5.7 & 1.23 \\
\hline 2-stage & 7.2 & 13.2 & 6.1 & 0.78 \\
\hline
\end{tabular}

Table 2. Quantitative evaluation on interactive detections with multiple clicks

\begin{tabular}{|l|c|c|c|c|c|c|c|c|c|}
\hline Number of user clicks & 2 & 3 & 4 & 5 & 6 & 7 & 8 & 9 & 10 \\
\hline False detection rate & $17.8 \%$ & $13.1 \%$ & $8.4 \%$ & $5.4 \%$ & $3.8 \%$ & $2.6 \%$ & $2.0 \%$ & $1.4 \%$ & $1.0 \%$ \\
\hline median (pixel error) & 6.51 & 2.29 & 1.25 & 0.99 & 0.95 & 0.92 & 0.91 & 0.88 & 0.87 \\
\hline mean (pixel error) & 7.58 & 3.72 & 2.06 & 1.36 & 1.19 & 1.07 & 1.06 & 0.96 & 0.94 \\
\hline std (pixel error) & 13.60 & 8.76 & 5.16 & 2.19 & 1.83 & 1.14 & 1.55 & 0.66 & 0.74 \\
\hline
\end{tabular}

user clicks. For a fair comparison, we select optimal parameters for each method. In our sensitivity test, the performance of all the methods are not sensitive to the parameter settings in a neighbor of optimal parameters. From Figure 6, it can be observed that the graph based methods perform better than the previous work [5], which gets an average error rate of $25 \%$ for the testing set containing diverse shapes. The method based on the hyper-graph, with an error rate of $17 \%$, has further improved from the original graph based method which has an error rate of $19 \%$. The computation time for the hyper-graph is slightly higher than that of the original graph. To get the advantage of accuracy from the hyper-graph and the advantage of efficiency from the graph, the two-stage algorithm gets the error rate of $17 \%$ with acceptable running time.

In the second experiment setting, multiple user clicks are provided. The users are asked to continuously provide clicks until the detection results are satisfactory. Table 2 summarizes the detection rates and pixel errors with multiple user clicks. The detection accuracy progresses with more user inputs. Usually after 4 clicks, the pixel error converges to less than 1 pixel. In the work $[5]$, its performance does not improve after 7 clicks, which means that the performance of their algorithm may reach its limits due to the lack of geometric constraints. The method can also be applied to detect other curve structures such as vessel, and catheter. Two examples are shown in Fig. [5. (b) and (c).

\section{Conclusion}

The paper provides a novel and practical method that can effectively and efficiently detect curve structures in 2D images. A novel graph based framework has been presented to combine automatically detected curve segments, user interactions, and geometric constraints for a smooth interactive detection. The method 
has been applied to the guidewire and vessel detections, and encouraging results have been obtained. The future work will be to apply the method to detect more types of curve structures, and to handle complex curve structures, such as loops.

\section{Acknowledgement}

We would like to thank Dr. Xun Xu for the discussions on the hyper-graph in Siemens Corporate Research.

\section{References}

1. Barbu, A., Athitsos, V., Georgescu, B., Boehm, S., Durlak, P., Comaniciu, D.: Hierarchical learning of curves application to guidewire localization in fluoroscopy. In: CVPR, Minneapolis, Minnesota, USA (2007)

2. Katz, N., Nelson, M., Goldbaum, M., Chaudhuri, S., Chatterjee, S.: Detection of blood vessels in retinal images using two-dimensional matched filters. IEEE Trans. Med. Imaging 8(3), 263-269 (1989)

3. Mortensen, E.N., Barrett, W.A.: Intelligent scissors for image composition. In: SIGGRAPH 1995: Proceedings of the 22nd annual conference on Computer graphics and interactive techniques, pp. 191-198. ACM, New York (1995)

4. Freedman, D., Zhang, T.: Interactive graph cut based segmentation with shape priors. In: CVPR, Washington, DC, USA, pp. 755-762. IEEE Computer Society, Los Alamitos (2005)

5. Mazouer, P., Chen, T., Zhu, Y., Wang, P., Durlak, P., Thiran, J.-P., Comaniciu, D.: User-constrained guidewire localization in fluoroscopy. In: Proc. SPIE of Medical Imaging: Image Processing (2009)

6. Viola, P., Jones, M.: Robust real-time object detection. International Journal of Computer Vision 57(2), 137-154 (2004)

7. Tu, Z.: Probabilistic boosting-tree: Learning discriminative models for classification, recognition, and clustering. In: ICCV, pp. 1589-1596 (2005)

8. Corme, T.H., Leiserson, C.E., Rivest, R.L., Stein, C.: Introduction to Algorithms, 2nd edn. MIT Press / McGraw-Hill (2001)

9. Diestel, R.: Graph Theory, 3rd edn. Springer, Heidelberg (2005)

10. Guy, G., Medioni, G.: Inferring global perceptual contours from local features. Int. J. Comput. Vision 20(1-2), 113-133 (1996) 